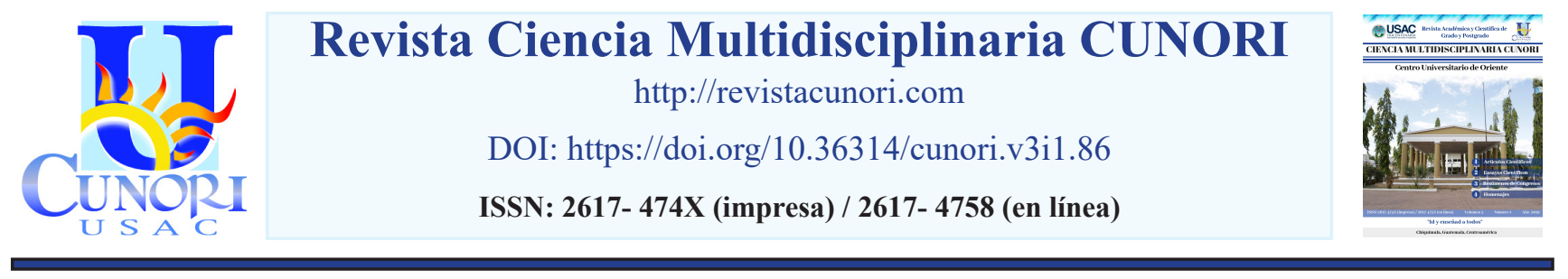

Como citar el artículo

Pereira, C., Barrios, A., Arriola, C., Retana, R., (2019). Caracterización epidemiológica y clínica de la hemorragia gastrointestinal superior. Revista Ciencia Multidisciplinaria CUNORI, 3(1), 109-116. DOI: https://doi.org/10.36314/cunori.v3i1.86

\title{
Caracterización epidemiológica y clínica de la hemorragia gastrointestinal superior
}

\section{Epidemiological and clinical characterization of upper gastrointestinal hemorrhage \\ Candida Pereira*, Ariel Barrios, Carlos Arriola, Ronaldo Retana \\ Centro Universitario de Oriente (CUNORI), Universidad de San Carlos de Guatemala}

Recibido: 31 de octubre de 2018 / Revisión: 28 de noviembre de 2018 / Aceptado: 15 de enero de 2019

Disponible en internet el 30 de agosto de 2019

Resumen

*Autor para correspondencia.

Correo electrónico: candidapereira.m@gmail.com

T a hemorragia gastrointestinal superior es denominada así a toda perdida sanguínea del tracto gastrointestinal por arriba del _ángulo de Treitz. Se realizo un estudio descriptivo retrospectivo, el cual caracterizo a 168 pacientes ingresados por hemorragia gastrointestinal superior ingresados al departamento de Medicina Interna del Hospital Nacional de Chiquimula en los meses de enero del año 2013 a diciembre del año 2017. La investigación fue realizada utilizando un instrumento de recolección de datos obteniendo como hallazgos, que el grupo etario con mayor predominio de presentación de morbilidad y mortalidad fue el encontrado en el rango entre $60-75$ años con $29 \%$ (49), con un ligero predominio del sexo masculino con $51 \%$ ( 85 ), y una prevalencia de 2 casos por cada 100 pacientes ingresados al departamento de Medicina Interna. Con respecto a las manifestaciones clínicas se pudo observar que las comunes fueron hematemesis con 79\% (133), anemia 71\% (120) y dolor abdominal 70\% (118). Se determino que las principales comorbilidades fueron alcoholismo con 40\% (68), HTA 23\% (39), DM 18\% (30) y hepatopatías 15\% (26) y entre los hábitos se encontraron el tabaquismo 22\% (37), uso de AINES 7\% (12) y ayuno prolongado 2\% (3). Se estableció que el tratamiento más utilizado fueron inhibidores de bomba específicamente omeprazol.

Palabras clave: hemorragia gastrointestinal superior, hepatopatía, antiinflamatorios, alcoholismo

Abstract

U pper gastrointestinal hemorrhaging is characterized as the total blood los of the gastrointestinal tract above the angle of Treitz. A study on this subject was done on 168 patients admitted for upper gastrointestinal hemorrhaging in the Internal Medicine departamento of the National Hospital in Chiquimula, Guatemala from january 2013 to december 2017. The investigation was performed using data obtained from evidence, and the age group with the largest morbidity rates was between 60-75 years old with an average of $29 \%$ (49). Of all the patients admitted, $51 \%$ (85) were male, with a frequency of 2 cases for every 100 patients admitted intto the Internal Medicine department. Whit respect to the clinical findings presented, it was observed that the predominant symptoms included hematemesis, with an average of $79 \%$ (133), anemia with 71\% (120) and abdominal pain with $70 \%$ (118). It was determine that the principal causes were alcoholism, with an average of $40 \%$ (68), hypertension with $23 \%$ (39), diabetes mellitus with 18\% (30) and hepatopathy with 15\% (26). The lifestyle habits found contributing to upper gastrointestinal hemorrhaging were smoking, with an average of 22\% (37), use of NSAIDs (nonsteroidal anti-inflammatory drugs) with 7\% (12) and prolonged fasting with $2 \%$ (3). The established treatment most prescribed was proton pump inhitors (PPIs); for example, omeprazole.

Keywords: upper gastrointestinal hemorrhaging, hepatopathy, anti-inflammatory drugs, alcoholism 


\section{Introducción}

La hemorragia gastrointestinal es la pérdida sanguínea provocada por una lesión que se origina en cualquier segmento del tubo digestivo; puede clasificarse según su localización en hemorragia gastrointestinal inferior (HGII) o hemorragia gastrointestinal superior (HGIS) estando la última delimitada por arriba del ángulo de Treitz (Villanueva et al 2016:55-85). Representa una de las condiciones clínicas más prevalentes en los servicios de urgencia hospitalaria y de gastroenterología (Taquirá \& García 2011:65). Las causas más frecuentes de hemorragia del tubo digestivo superior son las ulceropatías, gastroduodenitis. Otros orígenes son los relacionados con hipertensión portal, neoplasias, síndrome de Mallory Weiss, lesiones vasculares y varices de ellas las lesiones de origen péptico son su causa más frecuente (Hasler \& Owyang 2012: 2402-2408.).

Desde el punto de vista fisiopatológico la HGIS comparte muchas características hemodinámicas con el resto de hemorragias en el cuerpo como: la pérdida del volumen sanguíneo, shock hemorrágico, la hipoxemia tisular, alteración del gasto cardiaco, activación de la coagulación entre otras, pero así también existe una muy notable diferencia con todas ellas; y es que en la mayoría de las hemorragias en el resto del cuerpo, la sangre es lanzada hacia el exterior, y puede ser tratada de forma más convencional, mientras que los vasos afectos en el HGIS no son visibles, ni accesibles sin instrumentos especializados, aunado al hecho de que es muy frecuente la recidiva de la hemorragia y se encuentra asocia con una alta morbimortalidad (Colomo 2016:49).

Cuenta con una incidencia anual que oscila entre 50 y 150 casos por 100,000 habitantes, la incidencia resulta cada vez más alta en personas de mayor edad. Es más frecuente en hombres que en mujeres (IGSS 2011:69). Se conoce también que el porcentaje general de mortalidad fluctúa entre un 10\% y 36\% según diversas literaturas. En Guatemala, el número de hospitalizaciones por hemorragia gastrointestinal superior es elevado, teniendo una mortalidad representativa. Según el departamento de estadística del Hospital General San Juan de Dios en el año 2000 se atendieron aproximadamente 300 casos con una mortalidad del 47\%(Sagastume \& Cabrera 2016:79).

Actualmente no se cuenta en el área de Chiquimula con un registro previo sobre caracterización y prevalencia de la hemorragia gastrointestinal superior, sin embargo, es frecuente su atención en emergencias y servicios de centros hospitalarios, por lo cual se realizo un estudio descriptivo retrospectivo sobre la caracterización epidemiológica y clínica de 168 pacientes ingresados con HGIS al departamento de Medicina Interna del Hospital de Chiquimula durante los meses de enero del año 2013 a diciembre del año 2017. Este trabajo representa la primera fuente y guía de información al nivel del departamento de Chiquimula, sobre este tema, lo cual servirá posteriormente como base para la realización y seguimiento de más investigaciones relacionadas.

\section{Material y métodos}

Se revisaron $168(100 \%)$ de expedientes clínicos de pacientes ingresados al departamento de Medicina Interna del Hospital de Chiquimula, durante los meses de enero del año 2013 a diciembre del año 2017. 
Fueron incluidos todos aquellos pacientes de 12 años en adelante, tanto del sexo masculino como femenino y se excluyeron todos aquellos pacientes con ficha clínica incompleta al momento de la investigación. Se utilizó una boleta de recolección de datos que incluía datos generales del expediente, datos epidemiológicos como edad, sexo, ocupación y lugar de procedencia, y datos clínicos como: manifestaciones clínicas, forma de diagnóstico, comorbilidades, hábitos y tratamiento.

Posterior a la obtención de datos, se introdujeron en una base de datos de Windows (2013), se clasificaron de acuerdo a los diferentes objetivos de la investigación y se presentaron en forma de gráficas y formulas, todos ellos fueron utilizados para formar un perfil de pacientes con HGIS según el área geográfica de Chiquimula.

\section{Resultados}

\section{Morbi-mortalidad en pacientes}

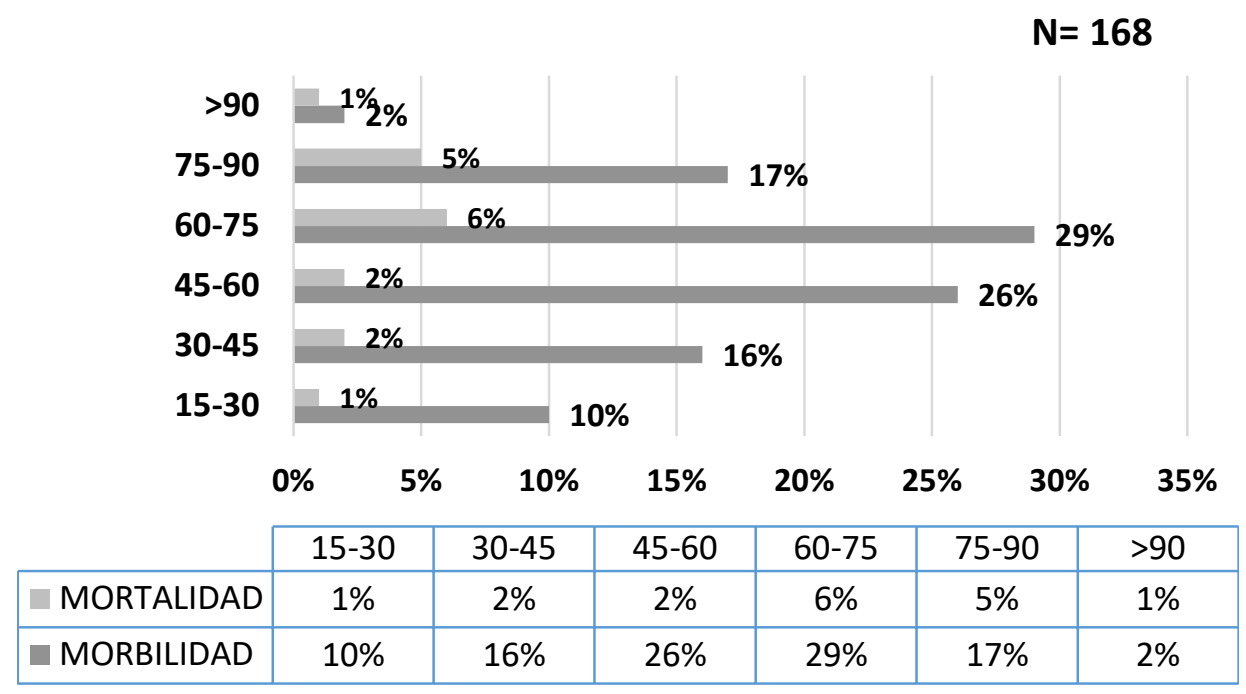

En la figura 1. De los 168 pacientes que forman el total de la población investigada, existe un mayor porcentaje de morbi-mortalidad en pacientes encontrados en el grupo etario de 60-75 años con $29 \%$ (49) de los pacientes y con un 6\%(10) de mortalidad. Con una prevalencia de 2 casos de hemorragia gastrointestinal superior por cada 100 ingresos al servicio de Medicina Interna. 


\section{Género de los pacientes}

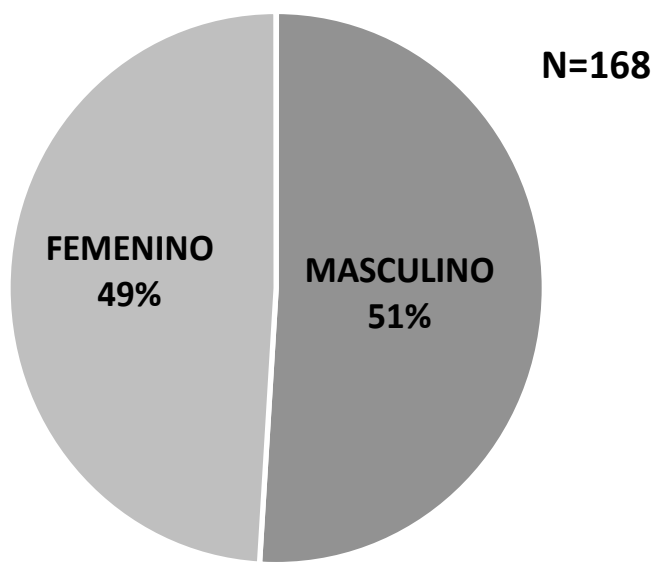

Figura 2. Se observa que del total de pacientes ingresados por hemorragia gastrointestinal superior se encontró que existe un predominio del sexo masculino con el 51\% (85), mientras que el 49\% (83) fueron del sexo femenino.

\section{Manifestaciones clínicas más comunes}

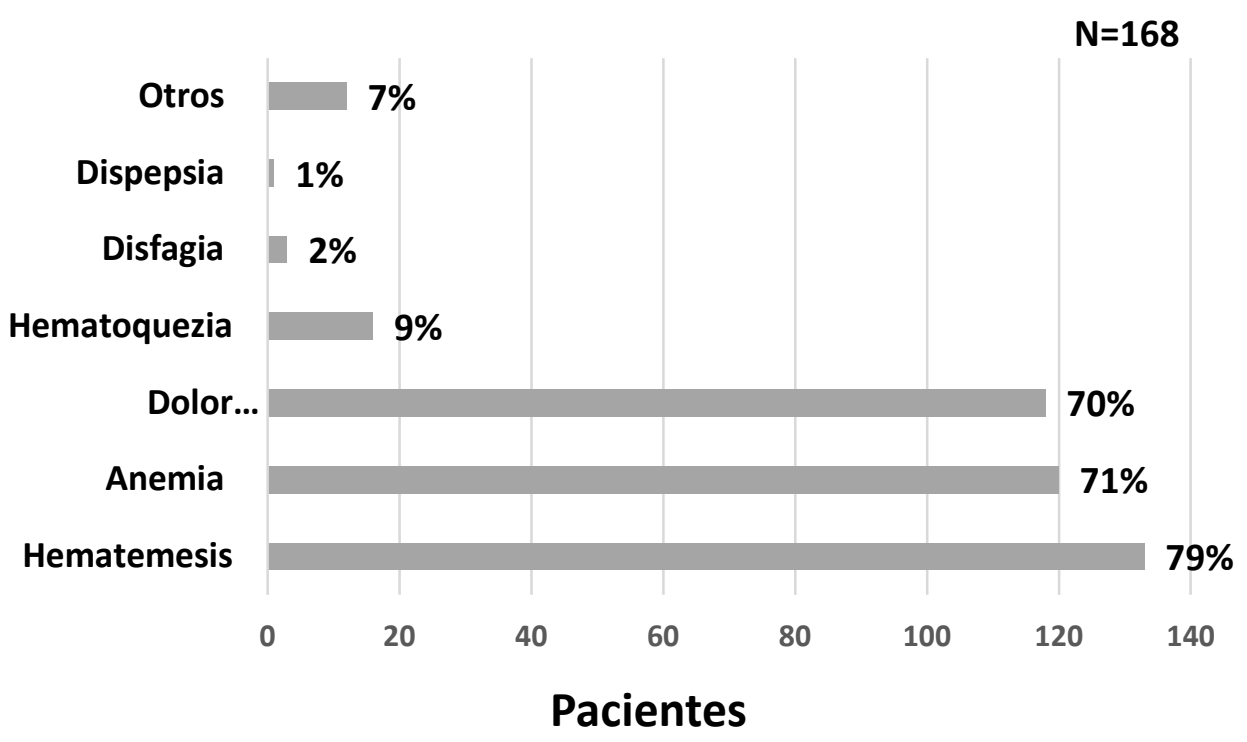

Figura 3. Se identificó que las manifestaciones clínicas más comunes fueron hematemesis con 79\% (133), seguido de anemia 71\% (120) y dolor abdominal 70\% (118); y dentro de los otros síntomas se encontraron: palidez, cansancio, alteración del estado de la conciencia, epistaxis, dolor precordial, dolor y edema de miembros inferiores. 


\section{Causas}

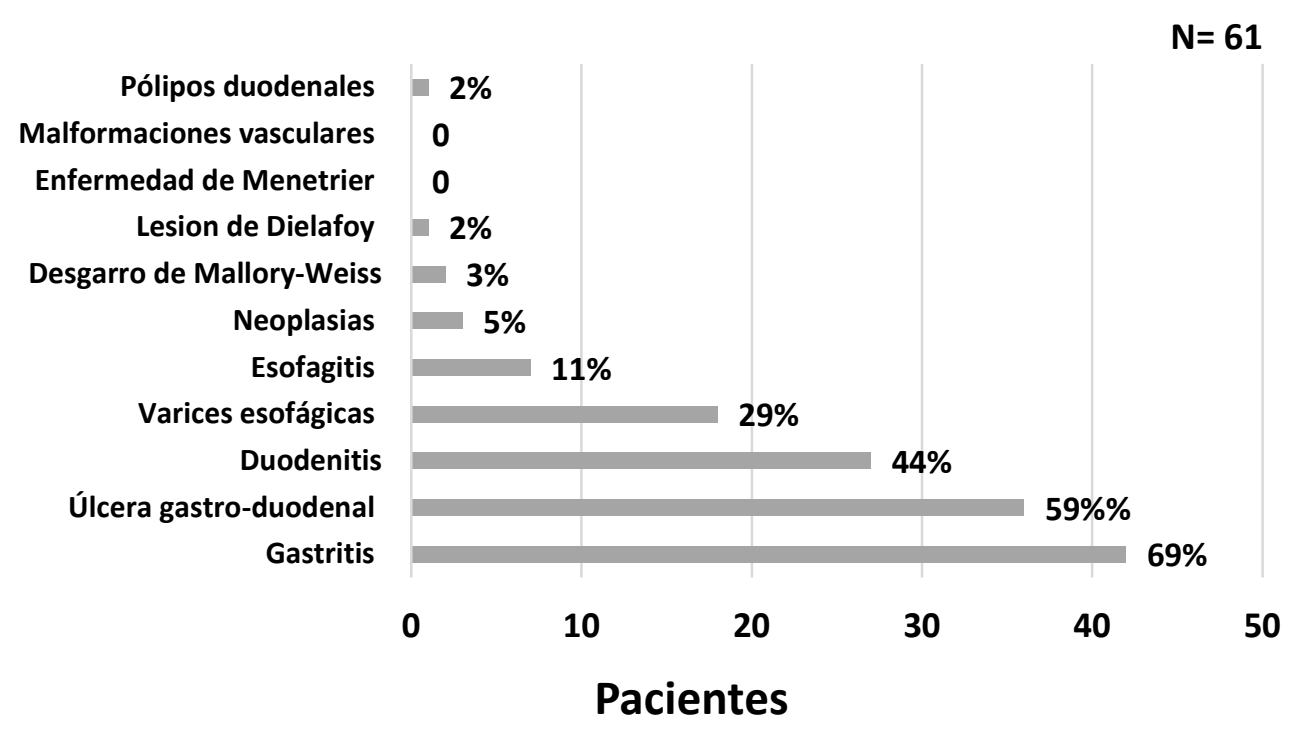

Figura 4. En cuanto a los hallazgos registrados y descritos en los informes de endoscopias, se observó el siguiente orden de frecuencia, según su causa no variceal: gastritis 69\% (42), ulceras gastro-duodenales $59 \%$ (36), duodenitis 44\% (27), esofagitis 11\% (7), entre otras causas. Y de causa variceal un total de $29 \%(18)$.

\section{Comorbilidades de los pacientes}

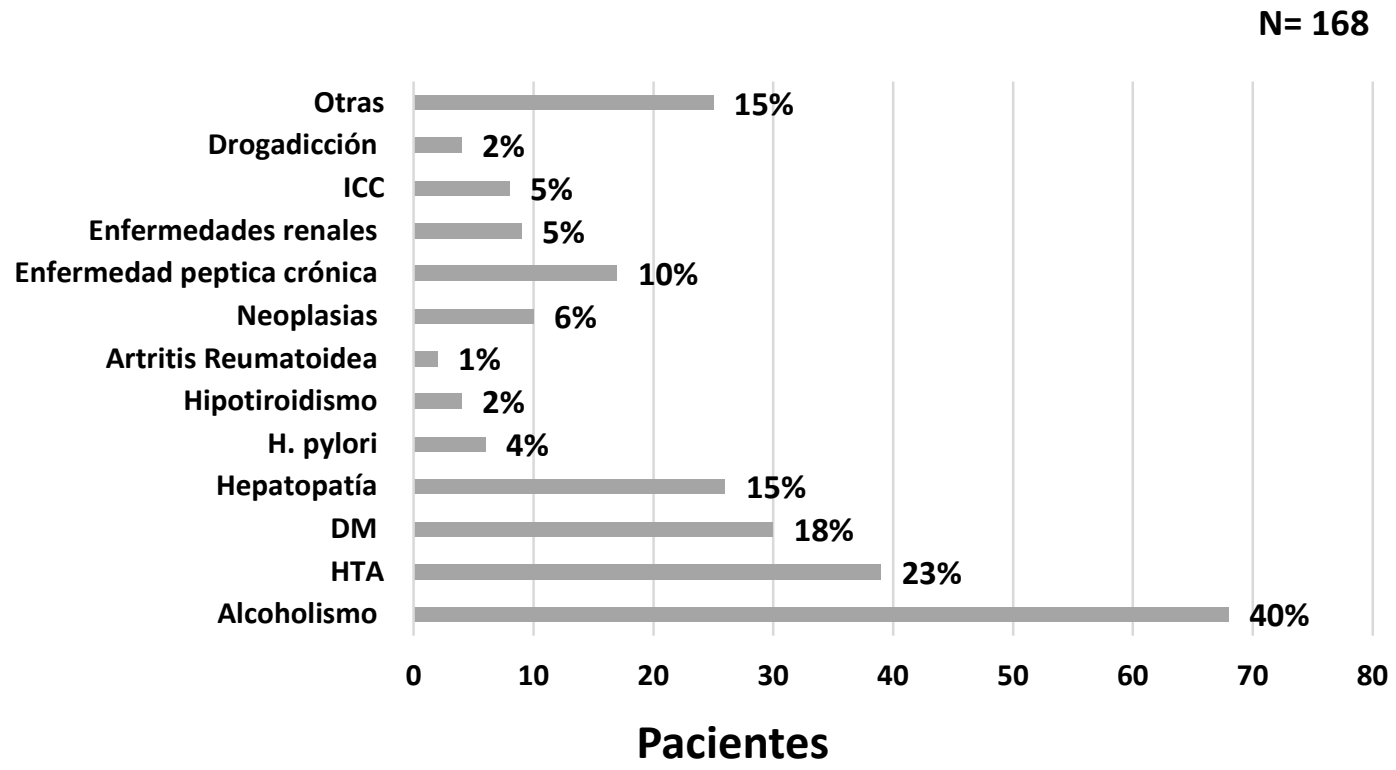

Figura 5. Al agrupar los datos se observó que el $92 \%$ (154) de pacientes presentaban comorbilidades de base dentro de las que más influencia tenía como factor de riesgo era alcoholismo con 40\% (68) de la población total, seguido por hipertensión arterial (HTA) con 23\% (39), diabetes mellitus (DM) con 18\% (30), aunado al número de hepatopatías crónicas descritas con 15\% (26). 


\section{Hábitos de los pacientes}

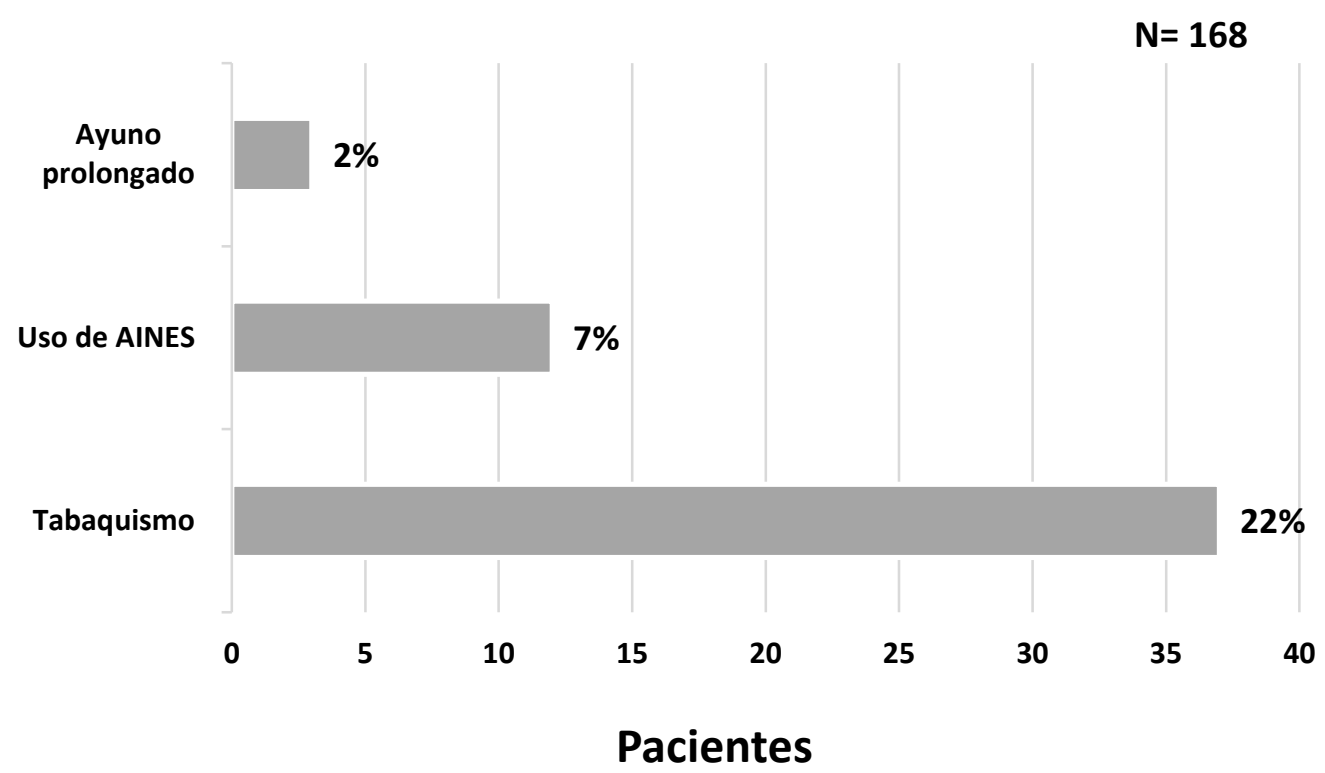

Figura 6. Se pudo observar que únicamente el 30\% (52) de los pacientes los presentaban hábitos, dentro de ellos, el que con mayor frecuencia presentaron fue tabaquismo con un total de $22 \%$ (37), y se observa una correlación de uso de antiinflamatorios no esteroideos (AINES) con la presencia de gastritis erosiva.

Con relación al tratamiento observamos que el 98\% (164) de pacientes recibió al menos un tipo de inhibidor de bomba, siendo el más utilizado Omeprazol con 96\% (162). En cuanto al uso de vasoconstrictores únicamente el 10\% (17) de pacientes de la población estudiada recibió tratamiento con Octreotide, sin haber hecho uso de ningún otro tipo de medicamento.

En referencia del uso de endoscopia como método de tratamiento el procedimiento utilizado fue ligadura con bandas elásticas con 8\% (5), y polipectomía 1\% (1) como tratamiento extraordinario por pólipo duodenal.

\section{Discusión}

El universo estuvo conformado por 168 pacientes ingresados al servicio de Medicina interna del Hospital de Chiquimula, con una prevalencia de 2 casos de hemorragia gastrointestinal superior por cada 100 ingresados al servicio de Medicina Interna, la proporción por sexo, presento un ligero predominio porcentual sin presentar ninguna significancia estadística por el sexo masculino con 51\% (85), respecto al sexo femenino con 49\% (83). La edad mínima de presentación de hemorragia gastrointestinal superior fue de 16 años 1\% (1) y la edad máxima fue de 92 años $2 \%$ (2). El grupo etario dentro de 60-75 años presento mayor morbilidad 29\% (49) y mayor mortalidad 6\% (10) de la totalidad de la población, lo que corresponde en comparación con lo descrito en las literaturas.

Se observó que de los pacientes fallecidos fueron un total del 17\%, el sexo fue mayoritariamente masculino con el $62 \%$ (18), en comparación con el sexo femenino con el 38\% (11). Respecto a las manifestaciones clínicas al momento del ingreso se observó que las más frecuentes presentadas fueron hemate- 
mesis con el 79\% (133) de los pacientes, seguido por anemia 71\% (120), y dolor abdominal 70\% (118), siendo menos frecuente dispepsia 2\% (2) y disfagia (1\%). Se realizó el diagnóstico de forma clínica en el 64\% (107) de los casos y únicamente un 36\% (61) de forma endoscópica.

En cuanto a los hallazgos endoscópicos descritos y registrados en el expediente clínico se encontraron que las causas de HGIS fluctúan de no variceal, siendo las más comunes gastritis 69\% (42), úlcera gastro-duodenales 59\% (36), duodenitis 44\% (27); y causa variceal 29\% (18), siendo la más común, la grado II 39\% (7), de las cuales únicamente el 28\% (5) de la totalidad de pacientes recibieron tratamiento endoscópico con ligadura de banda.

Con relación a los antecedentes patológicos se observó que la comorbilidad más presentada por los pacientes fue alcoholismo con 40\% (68), seguida por hipertensión arterial 23\% (39), diabetes mellitus 18\% (30) y hepatopatía crónica 15\% (26). Mientras que la presencia de hábitos se encontró que el tabaquismo 22\% (37), uso de antiinflamatorios no esteroideos AINES 7\% (12) que se encontraban relacionados con patologías como artritis, con un tiempo de uso mínimo de uso de 5 días y máximo de 3 años, y ayuno prolongado sufrido por el $2 \%$ (3) de los pacientes, los cuales indicaban se desarrollaban durante el uso prolongado de alcohol.

El tratamiento utilizado fue mayoritariamente inhibidor de bomba específicamente omeprazol con el 96\% (162) de la totalidad de la población sabiendo que estos se utilizan principalmente en HGIS no variceal para estabilización del coágulo y con respecto a los vasoconstrictores el único medicamento utilizado fue octreótide con un 10\% (17); y endoscópicamente fueron tratados 8\% (5) con ligadura con bandas elásticas y únicamente $2 \%$ (1) se le realizó polipectomía; como hallazgo de tratamiento también se encontró que el 53\% (89) recibió transfusiones sanguíneas.

\section{Referencias bibliográficas}

Colomo, A. (2016). Hemorragia digestiva alta: prevención y tratamiento. Barcelona, España, UAB. p.49. Disponible en https://ddd.uab.cat/pub/ tesis/2017/hdl_10803_400754/aco1de1.pdf.

Hasler, W. \& Owyang, C. (2012). Estrategias diagnósticas de las enfermedades del tubo digestivo. In Harrison: principios de medicina interna. 18 ed México, editorial Interamericana McGraw Hill. v.2.

Instituto Guatemalteco de Seguridad Social -IGSS. (2011). Guía de hemorragia gastrointestinal superior Guatemala, IGSS. p.69 (GPC-BE No. 33). Disponible en http://www.igssgt.org/images/gpcbe/ medicina_interna/GPC-BE\%2033\%20 HGS.pdf.

Sagastume, M. \& Cabrera, V. (2016). Caracterización clínica y endoscópica de la hemorragia gastrointestinal superior. Guatemala, USAC-CUNORI. p. 79. Disponible en http://cunori.edu.gt/descargas/ Caracterizacion_clinica_y_endoscopica_de_la_hemorragia_gastrointestinal_superior.pdf.

Taquirá, S. \& García, R. (2011). Caracterización epidemiológica y clínica de pacientes con diagnóstico de hemorragia gastrointestinal superior. Guatemala, USAC. p.65. Disponible en http://biblioteca. usac.edu.gt/tesis/05/05_8833.pdf. 
Villanueva, C., García, J. \& Hervas, A. (2016). Hemorragia gastrointestinal. Práctica clínica en gastroenterología y hepatología. España, AEG/Grupo CTO Editorial.Disponible en https://www.aegastro.es/ sites/default/files/files/03_Gastroenterologia.pdf.

\section{Sobre la autora}

\section{Candida Soledad Pereira Mellado}

Médico y Cirujano, egresada del Centro Universitario CUNORI, de la Universidad de San Carlos de Guatemala. Realizó su investigación con el título "Caracterización epidemiológica y clínica de la hemorragia gastrointestinal superior”, en el departamento de medicina interna del Hospital de Nacional de Chiquimula.

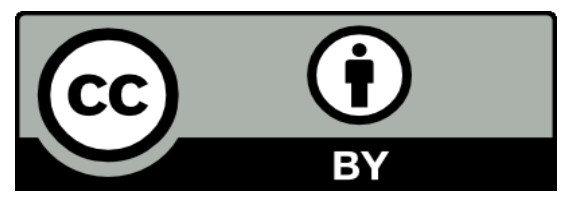

Este texto está protegido por una licencia CreativeCommons 4.0.

Usted es libre para compartir, copiar y redistribuir el material en cualquier medio o formato y adaptar el documento, remezclar, transformar y crear a partir del material para cualquier propósito, incluso comercialmente, siempre que cumpla la condición de atribución: usted debe reconocer el crédito de una obra de manera adecuada, proporcionar un enlace a la licencia, e indicar si se han realizado cambios. Puede hacerlo en cualquier forma razonable, pero no de forma tal que sugiera que tiene el apoyo del licenciante o lo recibe por el uso que hace. 